\title{
Antireflection coatings from analogy between electron scattering and spin precession
}

\author{
D. W. L. Sprunga) and Gregory V. Morozov \\ Department of Physics and Astronomy, McMaster University, Hamilton, Ontario L8S 4M1 Canada \\ J. Martorell \\ Departament d'Estructura i Constituents de la Materia, Facultat Física, University of Barcelona, \\ Barcelona 08028, Spain
}

(Received 24 September 2002; accepted 20 January 2003)

\begin{abstract}
We use the analogy between scattering of a wave from a potential, and the precession of a spin-half particle in a magnetic field, to gain insight into the design of an antireflection coating for electrons in a semiconductor superlattice. It is shown that the classic recipes derived for optics are generally not applicable due to the different dispersion law for electrons. Using the stability conditions we show that a Poisson distribution of impedance steps is a better approximation than is a Gaussian distribution. Examples are given of filters with average transmissivity exceeding $95 \%$ over an allowed band. (C) 2003 American Institute of Physics. [DOI: 10.1063/1.1559942]
\end{abstract}

\section{INTRODUCTION}

Antireflection coatings (ARC) are of great importance in many areas. In optics the aim is to maximize the transmission of visible light through lenses by applying coatings with suitable indices of refraction on both surfaces. For microwave transmission, the analogous problem is to minimize reflection at a junction between two sections of waveguide, by means of an impedance transformer consisting of sections of varying cross-section. Solutions for these problems were worked out in the 1950's and can be found in many books and reviews, of which we cite some representative examples. $^{1-6}$

Recently Gornik's group in Vienna ${ }^{7,8}$ have studied the analogous problem of constructing an energy bandpass filter for electrons in a semiconductor heterostructure. In a first paper they demonstrated a single-cell ARC, which raised the transmissivity through a superlattice to about $80 \%$ across the lowest-allowed band. In further work they proposed a double-cell device with even better properties. We have derived exact criteria for optimizing the properties of a singlecell ARC. ${ }^{9}$

The problem of constructing a passband filter for electrons has been discussed by several groups. Gaylord and collaborators ${ }^{10,11}$ very early wrote a series of papers proposing to take over the well-established solutions from optics and microwaves. Chang and Kuo ${ }^{12}$ translated the Gaylord approach into the language of impedance transformers. Tung and Lee ${ }^{13}$ and later Gomez et al. ${ }^{14}$ considered a rather different filter based on a Gaussian distribution of barrier strengths. Yang and $\mathrm{Li}^{15}$ extended this approach by using a variety of different distributions, but with no underlying theory as to why these might or might not work. In other words, previous approaches have either relied on the similarity to optical and microwave ARCs, assuming that this well-

\footnotetext{
${ }^{a)}$ Electronic mail: dwsprung@mcmaster.ca
}

developed theory would apply to electrons, or else they simply guessed at how to proceed.

The aim of the present article is threefold. First of all, we draw attention to an interesting analogy between (i) a particle scattering in a one-dimensional potential and (ii) a spin-half system precessing in a magnetic field. Second, we use this analogy to give a simple and intuitive explanation of how an impedance transformer, and consequently a bandpass filter, works. While such a picture does not entirely dispense with the ingenious calculations underlying the classical recipes for ARC cells and impedance transformers, it certainly provides insight to the crucial issues involved. In particular, it leads to the concept of stability conditions and their role in defining the parameters of an $N$-cell ARC.

Our third contribution is to identify the most important difference between bandpass filters for electrons and their counterparts in optics. This leads us to propose a simple model, called the linear model, which is similar to the true situation for electrons in semiconductor superlattices. We solve this model analytically, and show that the resulting filter is very different from the well-known Butterworth or binomial filter of optics or microwave engineering. As a practical application, our method is applied to the device of Pacher et al. and Coquelin et al. ${ }^{7,8}$ We find that their transmissivity could be significantly improved by following our method.

\section{TRANSFER MATRIX}

In the single-band envelope function approximation, the electron wave function satisfies the Schrödinger equation in a real potential and with an energy- and position-dependent (real) effective mass ${ }^{16} m^{*}(E, x)$

$$
\begin{aligned}
& \Psi(x, t)=e^{-i E t / \hbar} \psi(x) \\
& \frac{d^{2} \psi(x)}{d x^{2}}+\frac{2 m m^{*}}{\hbar^{2}}[E-V(x)] \psi(x)=0
\end{aligned}
$$




$$
k^{2}(x)=\frac{2 m}{\hbar^{2}} m^{*}(E, x)[E-V(x)] .
$$

The time-reversed spatial wave function is $\psi^{*}(x)$. Consider a potential cell, placed $-d<x<d$. Outside the cell, assuming zero potential, $k$ becomes constant and we may write the wave function in the form

$$
\begin{aligned}
& \psi(x)=a e^{+i k(x+d)}+b e^{-i k(x+d)}, \quad x \leqslant-d, \\
& =a^{\prime} e^{+i k(x-d)}+b^{\prime} e^{-i k(x-d)}, \quad x \geqslant+d .
\end{aligned}
$$

The amplitudes on opposite sides of a cell are related by

$$
\left(\begin{array}{l}
a \\
b
\end{array}\right)=\left(\begin{array}{ll}
M_{11} & M_{12} \\
M_{21} & M_{22}
\end{array}\right)\left(\begin{array}{l}
a^{\prime} \\
b^{\prime}
\end{array}\right),
$$

which defines our transfer matrix $M$. It has the properties $\operatorname{det} M=1$, and through $\operatorname{Tr} M=2 \cos \phi$, defines the Bloch phase associated with a periodic array of identical cells.

For a scattering problem with incident wave from the left, $a=1, b=r_{L}=r ; a^{\prime}=t_{L}=t, b^{\prime}=0$, one easily sees that the first column of $M$ is given by

$$
M_{11}=\frac{1}{t}, \quad M_{21}=\frac{r}{t} .
$$

The second column of $M$ is determined by conservation of flux, and by the corresponding equation for incident waves from the right, with amplitudes denoted $r_{R}=r^{\prime}, t_{R}$ $=t^{\prime}$. For a general potential, one can show that $t^{\prime}=t$, and $r^{\prime} / t^{\prime}=-r^{*} / t^{*}$. The result is

$$
M=\left(\begin{array}{cc}
\frac{1}{t} & -\frac{r^{\prime}}{t^{\prime}} \\
\frac{r}{t} & \frac{1}{t^{*}}
\end{array}\right)=\left(\begin{array}{cc}
\frac{1}{t} & \frac{r^{*}}{t^{*}} \\
\frac{r}{t} & \frac{1}{t^{*}}
\end{array}\right)
$$

without assuming parity invariance. Time reversal symmetry alone makes $M_{12}=M_{21}^{*}$ and $M_{11}=M_{22}^{*}$.

For a potential with reflection symmetry the additional property $r_{R}=r_{L}$ holds, which makes $M_{12}=-M_{21}=M_{21}^{*}$ pure imaginary. Following $\mathrm{Kard}^{3}{ }^{3}$ for a symmetric cell we can introduce a parametrization of $M$, valid in an allowed band

$M_{11}=\cos \phi-i \sin \phi \cosh \mu=M_{22}^{*}$,

$M_{21}=-i \sin \phi \sinh \mu=M_{12}^{*}, \quad$ where

$\cos \phi=\frac{1}{2} \operatorname{Tr} M=\operatorname{Re} M_{11}$, and $\tanh \mu=\operatorname{Im} M_{21} / \operatorname{Im} M_{11}$.

This form respects the relation $1 \leqslant 1 /|t|^{2}=1+\sin ^{2} \phi \sinh ^{2} \mu$ as well as $\operatorname{det} M=1$, but applies only in an allowed miniband where the Bloch phase $\phi$ is real. ${ }^{17}$ We call $\mu$ the impedance parameter, because in the case of a square well, $e^{\mu}$ is the impedance, the ratio of velocity outside to inside the well. ${ }^{12}$

For an arbitrary cell, a different phase $\beta$ occurs on the off-diagonal elements

$$
M_{21}=-e^{i \beta} \sin \phi \sinh \mu=M_{12}^{*} .
$$

The symmetric cell corresponds to the special case $\beta$ $=+\pi / 2$. For the moment we confine our attention to symmetric cells for which we write

$$
\begin{aligned}
M & =\cos \phi\left(\begin{array}{ll}
1 & 0 \\
0 & 1
\end{array}\right)-i \sin \phi\left(\begin{array}{cc}
\cosh \mu & -\sinh \mu \\
\sinh \mu & -\cosh \mu
\end{array}\right) \\
& =\cos \phi \mathbf{I}-i \sin \phi U(2 \mu),
\end{aligned}
$$

with $U(2 \mu)=\cosh \mu \sigma_{z}-i \sinh \mu \sigma_{y}=\sigma \cdot \vec{n}$,

where $\vec{n}$ is a (complex) unit vector in the $Y Z$ plane. The meaning of this becomes evident if we write $\mu=i \eta$, giving

$$
M=e^{-i \phi \sigma_{n}}=R_{n}(2 \phi),
$$

where $\sigma_{n}=\sigma \cdot \mathbf{n}=\cos \eta \sigma_{z}+\sin \eta \sigma_{y}$.

We recognize $R_{n}$ as the operator which rotates a spin-1/2 system by angle $2 \phi$ around the axis $\mathbf{n},{ }^{18}$ which in this instance lies in the $Y Z$ plane. For asymmetric cells, the axis of rotation has azimuthal angle $\beta$. The axis of rotation is imaginary, but this does not invalidate the analogy.

Now consider an array of cells which need not be identical. The transfer matrix for the $p$ th cell is of the same form as Eq. (8), with parameters $\phi_{p}$ and $\mu_{p}=i \eta_{p}$. It can be factorized as follows:

$$
\begin{aligned}
M_{p} & =e^{+i\left(\eta_{p} / 2\right) \sigma_{x}} e^{-i \phi_{p} \sigma_{z}} e^{-i\left(\eta_{p} / 2\right) \sigma_{x}} \\
& \equiv Y\left(\mu_{p}\right) P\left(\phi_{p}\right) Y\left(-\mu_{p}\right) .
\end{aligned}
$$

$Y(\mu)$ is associated with a step-up in impedance from zero to $\mu$. The factors can be interpreted as follows. The top line is a rotation operator acting to the right on a ket. The spin-half system is rotated around $\mathrm{OX}$ by angle $\eta_{p}$, so an axis inclined initially at angle $\eta_{p}$, lines up along OZ. Then the system is rotated by angle $\phi_{p}$ around $\mathrm{OZ}$, and finally rotation around $\mathrm{OX}$ by angle $-\eta_{p}$ restores the axis to its initial position. The net effect is a rotation of the whole system by angle $\phi_{p}$ around the axis of rotation oriented at polar angle $\eta_{p}$. In the second line, a system consisting of left/right moving waves is acted on by a transfer matrix. The first factor lowers the impedance by $\mu_{p}$; then it propagates freely accumulating phase $\mp \phi_{p}$ on the upper/lower components, and finally the impedance is restored to its original value. The net effect is the same as propagating at an average impedance $\mu_{p}$ and accumulating the same phase.

In this analogy, a wave traveling to the right, outside the array, corresponds to a spin-up (along OZ) state, and a wave traveling to the left, to a spin-down state. When a rightmoving state encounters a potential, it is partly transmitted and partly reflected. Analogously a spin-up state placed in a magnetic field oriented at polar angle $\eta$ will precess around the field direction, thereby acquiring some spin-down (reflected wave) component. For a symmetric potential cell, the magnetic-field direction lies in the $Y Z$ plane. For a general cell, the polar angle is the same, but the azimuthal angle is $\beta$ : The asymmetric system differs only by a rotation around OZ. 


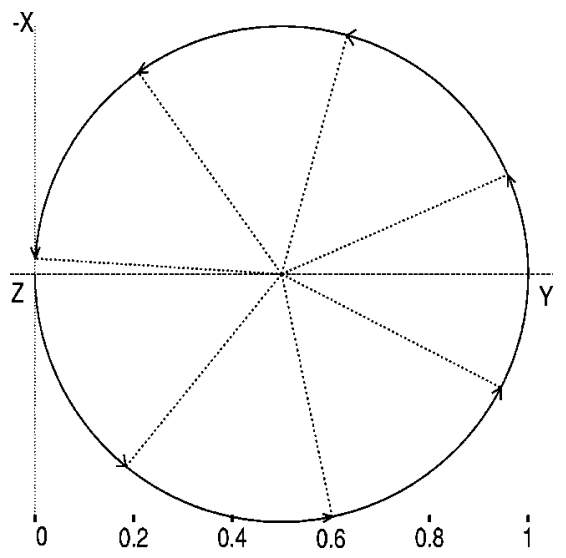

FIG. 1. Pseudospin path viewed from above for a periodic system with $\mu_{X}=0.5, N=7$.

This analogy is very helpful in seeing how a system will respond to a sequence of potential cells. For identical cells $\eta_{p}=\eta ; \phi_{p}=\phi$, the rotations are all about the same axis. The spin precesses on a cone whose polar angle is $\eta$; the cone intersects the sphere in a circle. Viewed from above the unit sphere, the spin moves on a circle centered at $\eta$. If there are $N$ such cells, the total angle of rotation will be $2 N \phi$. The condition for perfect transmission is that the spin state be returned to lie along OZ; this requires $2 N \phi=2 \pi m$ where $m$ is an integer. Within an allowed band, the Bloch phase increases by $\pi$, so the possible values are $m=1,2, \ldots, N-1$. An array of $N$ identical cells will show $N-1$ narrow resonances in each allowed band. ${ }^{19}$ They are narrow, for when the energy is varied slightly, changing $\phi \rightarrow \phi-\varepsilon$, the total phase changes by $2 N \varepsilon$, which quickly moves off the resonance condition. This situation is illustrated in Fig. 1 for an array of seven cells, and for $m=1$; the phase angle is off resonance by $1 \%$. One is looking down on the surface of the sphere from a point above the center of the circle. The radial lines mark off sectors of angular width $2 \phi \approx 2 \pi / 7$.

If the cells are not identical, but the impedance parameters $\eta_{p}$ are close, then the sequence of rotations will be on arcs of circles whose centers jump about. So long as these jumps are small compared to the radii of the circles, the total angle of rotation will be just twice the sum of the angles $\phi_{p}$. The behavior of the array will then be similar to a strictly periodic array. It will still wind $N$ times around a closed path, coming more or less close to the origin (at OZ) once on each traversal. We will have similar behavior to the case of identical cells, but with the sum of the $\phi_{p}$ playing the role of $N \phi$. This is the "mean phase lemma."

Conversely, if the $\eta_{p}$ are increasing rapidly, so the center of each rotation lies outside the circle of the previous one, then the path followed on the surface of the unit sphere will not wind more than once around the circumference. We will see that this topologically very different behavior is characteristic of impedance transformers and filters.

\section{QUARTER-WAVE IMPEDANCE TRANSFORMER}

In this section we will show that the criteria for quarterwave filters can be easily understood from our spin analogy. Further we will show that the classic recipes do not apply to the semiconductor case unless significant modifications are made. These lead to the conclusion that a Poisson distribution of the impedance steps between cells is more appropriate than is a Gaussian recipe.

For an electron in the conduction band, a layered heterostructure acts as a series of nonoverlapping potential cells. A cell can contain any number of homogeneous layers, or may even be continuously graded. No matter how complicated the potential (or the effective mass) may be, the scattering properties of a single cell are described by just two (or three) energy-dependent parameters $\phi, \mu$ (and $\beta$ ).

As discussed in Ref. 9, the first step in designing an energy bandpass filter is to find a cell with an allowed miniband covering the desired energy range. If it is the lowestallowed band, then $\cos \phi\left(E_{\ell}\right)=1$ and $\cos \phi\left(E_{u}\right)=-1$ at the lower- and upper-band edges. By placing a number $K$ of such cells together, a well-defined miniband will be obtained with essentially zero transmission outside the allowed band. If the cell is symmetric, any number of them will also be reflection symmetric, and the transfer matrix $M_{X}^{K}$ for this array will be described by just two parameters, $\mu_{X}$ and $\phi_{X}$.

An ARC consists of an additional potential $v_{A}(x)$ placed on one side of $X$ and its reflection $v_{A}^{\pi}(x)$ on the other side. The corresponding transfer matrices will be denoted $A$ and $A^{\pi}$. According to the spin analogy, $A$ rotates the spin by angle $2 \phi_{A}$ about an axis specified by its impedance parameters $\mu_{A}$ and $\beta_{A}$. For simplicity consider the case where both $X$ and $A$ are symmetric cells. Then the axes of rotation both lie in the $Y Z$ plane. At an arbitrary energy in the allowed band, if $A$ rotates the initial spin-up state by angle $\pi$, it will be converted to a state whose spin is oriented along another radius in the $Y Z$ plane. By choosing $\mu_{A}=\mu_{X} / 2$, the new orientation of the spin state will coincide with the direction $\mu_{X}$. Passing through the potential cells $X$ alters the spin state only by a phase factor $\mp \phi_{X}$, because the new state is an eigenstate of spin along this direction.

Rotation by angle $\pi$ means that $\phi_{A}=\pi / 2$, so that the ARC cell $v_{A}$ must be a Bragg reflector at the desired energy. This is the solution obtained in Ref. 9. The downstream ARC cell $A^{\pi}$ then rotates the spin state back to lie along OZ, representing a wave moving purely to the right, and giving perfect transmission. The path followed by the pseudospin state is illustrated in Fig. 2(a). It does not matter how many cells of type $X$ there are, because once the state is aligned along the direction $\mu_{X}$ it is in a spin eigenstate along that direction, and precession gives just an overall phase, which does not alter the probability of being in a spin-up or -down state along OZ. Such a state is a scattering eigenstate for the potential $v_{X}$.

An ARC may consist of more than one cell, for example two as illustrated in Fig. 2(b), or three in Fig. 2(c). In the general case we will number the cells $1,2, \ldots, N$ on the left, with the reflected ordering on the right. Let $M_{X}$ be the transfer matrix for the central cells, (the original system). Then, using the representation of Eq. (10), the total transfer matrix can be written in two equivalent forms

$$
\begin{aligned}
M_{T}=A M_{X} A^{-1 *} & =A Y\left(\mu_{X}\right) P\left(\phi_{X}\right) Y\left(-\mu_{X}\right) A^{-1 *} \\
& \equiv M P\left(\phi_{X}\right) M^{-1 *} .
\end{aligned}
$$




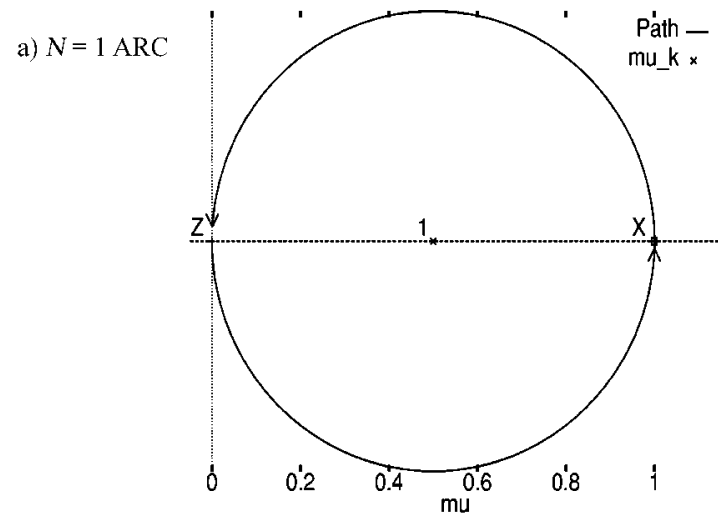

b) $N=2$ ARC

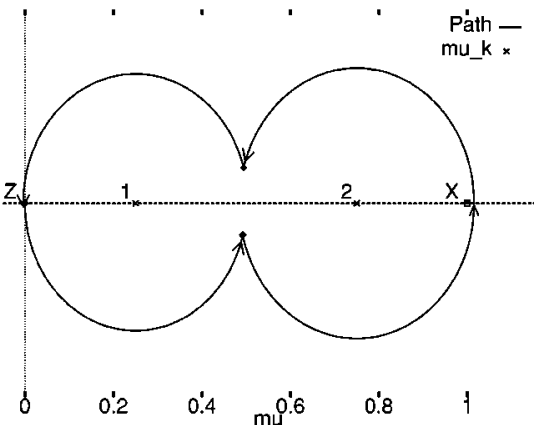

c) $N=3 \mathrm{ARC}$

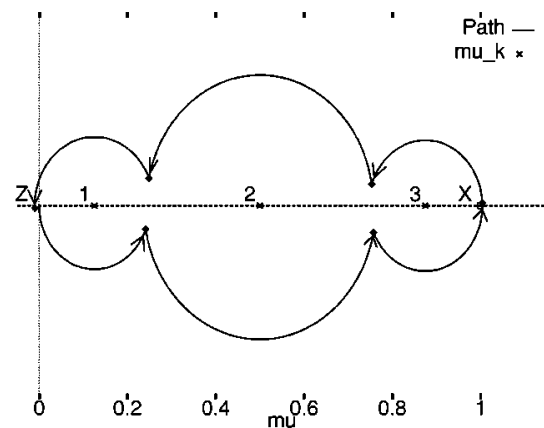

FIG. 2. Pseudospin paths for binomial ARCs. The $\phi_{k}$ are off the Bragg point by (a) $1 \%$, (b) $8 \%$, and (c) $12 \%$. The $\mu_{k}$ are in units of $\mu_{X}$.

In the first form, $A$ represents an impedance transformer, which takes the incident plane wave and prepares it to propagate through the central cells, described by $M_{X}$.

In the second form, $A$ is combined with a step-up operator $Y\left(\mu_{X}\right)$ which undoes this, rotating the state back to the OZ axis, so that the propagator $P\left(\phi_{X}\right)$ need only supply the phase factors $\mp \phi_{X}$ as the wave propagates through the central cells. In detail, $M$ consists of

$$
M=M_{1} M_{2} \cdots M_{N} Y\left(\mu_{X}\right) .
$$

According to the spin picture, when $M$ acts on a state $|+, Z\rangle$ which is "spin-up" along OZ, it has to leave it in the same condition. This requires that the element $M_{21}=0$. The reflected operator $M^{-1 *}$ performs the inverse rotations, restoring the state to be spin-up along OZ. It is sufficient to construct $M$ in order to make a passband filter. Incidentally, this proves that the design of a passband filter, at the design energy, does not involve the Bloch phase $\phi_{X}$ of the central cells. While it may appear complicated to combine $Y$ along with $A$, it is actually a big simplification, because each of the transfer matrices $M_{k}$ needs to be considered only once, not twice as would be the case if we worked with $M_{T}$.

In the classic designs, each cell of a multicell ARC is reflection symmetric, so $M$ and $M^{-1 *}$ differ only in the reverse ordering of the cells, and replacement of the step-up impedance factor $Y\left(\mu_{X}\right)$ by a step-down, $Y\left(-\mu_{X}\right)$. In this case, the simplest solution is to make each cell into a Bragg reflector at the design energy, with $\phi_{p}=\pi / 2$. Such a solution was illustrated in Fig. 2(b), for $N=2$ cells. The question arises, what possible advantage can come from using two cells as opposed to a single cell? The answer is found by supposing that the energy is varied by a small amount, so that $\phi_{p} \rightarrow \pi / 2-\varepsilon_{p}$. Then on the first rotation, the spin does not quite reach the axis. In the small angle approximation, the second rotation has a head start by $2 \varepsilon_{1}$, and it will land within $2\left(\varepsilon_{2}-\varepsilon_{1}\right)$ of the point $\mu_{X}$. If the phases $\phi_{p}$ are in fact equal, then the two deviations cancel out, and the $N$ $=2$ filter will have first-order stability. Since the single-cell filter has no such compensation available, it will go off resonance as soon as the energy varies from the design energy. The two-cell filter goes off resonance only when the squares $\varepsilon_{p}^{2}$ become significant.

The situation of equal Bloch phases in every cell generally applies in optics or microwaves, because (for normal incidence) the phase accumulated in passing through a cell is just $\phi_{p}=k_{0} n_{p} a_{p}$, the product of wave number in vacuum, the index of refraction, and the cell thickness. (Usually a cell is a single homogeneous layer in optics.) If the $\phi_{p}$ are arranged to be equal at the common Bragg point, then they will remain equal so long as the index of refraction is constant. This is not the case however in semiconductors because the wave-number $k(x)$ is the square root of an energy difference, as in Eq. (1).

For $N=3$ cells the classic Butterworth filter design has $\mu_{1}=1 / 8 \mu_{X}, \mu_{2}=1 / 2 \mu_{X}$, and $\mu_{3}=7 / 8 \mu_{X}$. In units of $\mu_{X}$, the rotations of the spin analogy have radii $1 / 8,1 / 4$, and $1 / 8$, as illustrated in Fig. 2(c). It is not hard to see that this model also shows linear stability as the energy varies from the multi-Bragg point, with a deviation in angle of twice $\varepsilon_{1}$ $-2 \varepsilon_{2}+\varepsilon_{3}$ which vanishes when the $\varepsilon_{p}$ are all equal. Moreover, this design also exhibits quadratic stability, defined as second order in the $\varepsilon_{p}$. In other words, the zero of the reflection amplitude at the design energy will be a secondorder zero, leading to a flatter maximum in transmission. Comparing the three panels of Fig. 2, one sees that the Bloch phases $\phi_{k}$ can be further off the Bragg point when $N$ is larger, and the device can still give very good transparency.

\section{ANALYTICAL DEVELOPMENT}

At this point it is useful to derive and extend the above results analytically. From Eq. (10), for each symmetric cell included in the ARC transfer matrix $M$, we can write

$$
M_{p}=-i \sin \phi_{p}\left[U\left(2 \mu_{p}\right)+i \cot \phi_{p} \mathbf{I}\right] .
$$

For brevity, we will write $U_{p}$ for $U\left(2 \mu_{p}\right)$. At the design energy, every $\phi_{p}=\pi / 2$, and $M$ reduces to a product of $U$-matrices. This product is easily reduced because the multiplication table for the $U$ and $Y$ matrices is very simple: 


$$
\begin{aligned}
& U(\mu)=\left(\begin{array}{lr}
\cosh \mu / 2 & -\sinh \mu / 2 \\
\sinh \mu / 2 & -\cosh \mu / 2
\end{array}\right), \\
& Y(\mu)=\left(\begin{array}{lr}
\cosh \mu / 2 & \sinh \mu / 2 \\
\sinh \mu / 2 & \cosh \mu / 2
\end{array}\right)=e^{(\mu / 2) \sigma_{x},} \\
& U_{a} U_{b}=Y\left(\mu_{a}-\mu_{b}\right), \quad U_{a} Y_{b}=U\left(\mu_{a}-\mu_{b}\right), \\
& Y_{a} U_{b}=U\left(\mu_{a}+\mu_{b}\right), \quad Y_{a} Y_{b}=Y\left(\mu_{a}+\mu_{b}\right) .
\end{aligned}
$$

Like matrices give a $Y$; unlike give a $U$. If a $Y$ is to the left we get the sum of arguments, and if a $U$, the difference. Therefore at the Bragg point we can reduce the product as follows:

$$
\begin{aligned}
(i)^{N} M & =\prod_{p=1}^{N} U\left(2 \mu_{p}\right) Y\left(\mu_{X}\right) \\
& =Y\left(2 \mu_{1}-2 \mu_{2}\right) \prod_{p=3}^{N} U\left(2 \mu_{p}\right) Y\left(\mu_{X}\right) \\
& =U\left(2 \mu_{1}-2 \mu_{2}+2 \mu_{3}\right) \prod_{p=4}^{N} U\left(2 \mu_{p}\right) Y\left(\mu_{X}\right) \cdots .
\end{aligned}
$$

If $N$ is even we arrive at the penultimate step with a $Y$ whose argument is an alternating sum of $\mu$ 's, ending with $-2 \mu_{N}$. The last multiplication uses the rule for $Y \times Y$, which adds $\mu_{X}$. When $N$ is odd, we end up with a $U$, whose argument ends with $+2 \mu_{N}$, and the $U \times Y$ product gives a $Y$ whose argument (denoted $\mu_{\Sigma}$ ) ends with $2 \mu_{N}-\mu_{X}$. In either case, the matrix element is $M_{21}=\sinh \mu_{\Sigma}$, and the condition for zero reflection is that $\mu_{\Sigma}=0$. Explicitly,

$$
\mu_{\Sigma}=\sum_{p=1}^{N}(-)^{p+1} 2 \mu_{p}+(-)^{N} \mu_{X} .
$$

All the equations we will deal with are simpler when written in terms of the steps in $\mu$, (including $\mu_{0}=0$ ), which we define as $\Delta_{p}=\mu_{p+1}-\mu_{p}$. Then we can write

$$
\mu_{\Sigma}=\sum_{p=0}^{N}(-)^{p} \Delta_{p}=0
$$

while

$$
\mu_{X}=\sum_{p=0}^{N} \Delta_{p} .
$$

For a single-cell ARC, $N=1$, the solution is $\Delta_{1}=\Delta_{0}$ $=\mu_{X} / 2$, so $\mu_{1}=\mu_{X} / 2$. In terms of indices of refraction, for the optical case this is the well-known solution $n_{1}=\sqrt{n_{X} n_{0}}$.

For more than one cell these two conditions are not sufficient to select a unique solution. A solution can be written down by considering the function

$$
F_{N}(x)=\sum_{p=0}^{N} x^{p} \Delta_{p}
$$

with

$$
F_{N}(1)=\mu_{X} ; \quad F_{N}(-1)=\mu_{\Sigma}=0
$$

The Butterworth ${ }^{6}$ or binomial solution makes $F_{N}(x)$ proportional to $(1+x)^{N}$, by setting

$$
\Delta_{p}=\frac{\mu_{X}}{2^{N}} C_{p}=\Delta_{N-p}
$$

So it is the steps, not the impedances themselves, which are the simple quantities. The $N=2$ and 3 filters drawn earlier are of this type. The solution is plausible if you interpret $x$ $=e^{2 i \phi}$ as being the same for every cell, and taking the value -1 at the multi-Bragg point. Then the matrix element $M_{21}$ will have an $N$ th order zero there.

Even for moderately large $N$, the limit of the binomial distribution is a Gaussian. With the steps $\Delta_{p}$ obeying a Gaussian law, the $\mu_{p}$ will be distributed like the error function. This may be the basis for the folklore that a Gaussian distribution of barrier heights should be associated with ARC cells.

\section{STABILITY CONDITIONS}

For small deviations from the multi-Bragg point, we write $\phi_{p}=\pi / 2-\varepsilon_{p}$, and in Eq. (13), the $\cot \phi_{p}$ becomes $\tan \varepsilon_{p} \equiv t_{p}$ for short. We treat the $t_{p}$ as small quantities, and derive stability conditions which involve $m$ of them at a time.

Using the representation Eq. (13) in the product of Eq. (12), and grouping terms with the same number of $t_{p}$, the general form of the transfer matrix becomes

$$
\begin{aligned}
M= & \prod_{p=1}^{N}\left(-i \sin \phi_{p}\right)\left[\prod_{p=i}^{N} U_{p}+i \sum_{k=1}^{N} t_{k} \prod_{p \neq k} U_{p}\right. \\
& -\sum_{k<m} t_{k} t_{m} \prod_{p \neq k, m} U_{p}-i \sum_{k<m<r} t_{k} t_{m} t_{r} \prod_{p \neq k, m, r} U_{p} \\
& +\cdots] Y\left(\mu_{X}\right) .
\end{aligned}
$$

Using the multiplication table for the matrices $U$ it is straightforward to write down the terms of any order. The leading term involves no $t_{k}$. Then there are sets of terms which involve $1,2,3, \ldots$, of the $t_{k}$. The first $N-1$ of these sets give stability conditions which must be imposed to make the resulting transmission amplitude as flat as possible in the region around the multi-Bragg point. The last term involves the product of all the $t_{k}$ and is simply

$$
M=Y\left(\mu_{X}\right) \prod_{p=1}^{N} \cos \phi_{p} .
$$

This is what remains when all the stability conditions have been satisfied, and is the generalization of the Butterworth filter for unequal phases $\phi_{p}$.

The linear stability terms are obtained by including one of the $t_{k}=\tan \varepsilon_{k}$ in place of the factor $U_{k}$ in the product Eq. (12). The typical contribution is

$$
\delta^{(1)} M \sim U_{1} \ldots U_{k-1} t_{k} U_{k+1} \ldots U_{N} Y\left(\mu_{X}\right),
$$


TABLE I. Equations which determine the $\Delta_{k}$ for the $N=7$ filter, in the optical case.

\begin{tabular}{ccccccccc}
\hline \hline$\Delta_{0}$ & $\Delta_{1}$ & $\Delta_{2}$ & $\Delta_{3}$ & $\Delta_{4}$ & $\Delta_{5}$ & $\Delta_{6}$ & $\Delta_{7}$ & $=$ value \\
\hline 7 & -6 & 5 & -4 & 3 & -2 & 1 & & 0 \\
& -6 & 10 & -12 & 12 & -10 & 6 & & 0 \\
35 & -20 & 15 & -16 & 19 & -20 & 15 & & 0 \\
& -20 & 20 & -16 & 16 & -20 & 20 & & 0 \\
21 & -6 & 11 & -12 & 9 & -10 & 15 & & 0 \\
& -6 & 2 & -4 & 4 & -2 & 6 & & 0 \\
1 & 0 & 1 & 0 & 1 & 0 & 1 & 0 & $\mu_{X} / 2$ \\
0 & 1 & 0 & 1 & 0 & 1 & 0 & 1 & $\mu_{X} / 2$ \\
\hline \hline
\end{tabular}

where $U_{p}=U\left(2 \mu_{p}\right)$. Because $U_{k}$ is missing from the product, the result of these multiplications is to arrive at either a $U$ or $Y$ matrix whose argument differs from $\mu_{\Sigma}$ in two respects: (i) the argument $2 \mu_{k}$ is missing and (ii) the terms following $\mu_{k-1}$ have the wrong sign as compared to $\mu_{\Sigma}$. The typical case is

$$
\begin{aligned}
2 \mu_{1} & -2 \mu_{2}+\cdots+2 \mu_{k-1}-2 \mu_{k+1} \cdots \\
& =-\mu_{0}+2 \mu_{1}-\cdots+2 \mu_{k-1}-\mu_{k}+\mu_{k}-2 \mu_{k+1} \cdots \\
& =\Delta_{0}-\Delta_{1}+\cdots-\Delta_{k-1}-\Delta_{k}+\Delta_{k+1}+\cdots
\end{aligned}
$$

which is to be compared with $\mu_{\Sigma}$ :

$$
0=\Delta_{0}-\Delta_{1}+\cdots-\Delta_{k-1}+\Delta_{k}-\Delta_{k+1}+\cdots .
$$

The contribution of this term to $M_{21}$ involves a factor $t_{k}$ times the sinh of this argument. Again the aim is to make the 2,1 matrix element vanish, which means that the sum of all these contributions must be zero. We can simplify the argument of the sinh by adding to it $\mu_{\Sigma}$ which is already zero, (the last line above). Then the terms following $\Delta_{k-1}$ cancel out. As a result we can write the linear stability condition as follows:

$$
\begin{aligned}
\delta^{(1)} M= & \sum_{k=1}^{N} t_{k} \sinh \left[\sum_{p=0}^{k-1}(-)^{p} \Delta_{p}\right]=0 \\
= & t_{1} \sinh \Delta_{0}+t_{2} \sinh \left(\Delta_{0}-\Delta_{1}\right) \\
& +t_{3} \sinh \left(\Delta_{0}-\Delta_{1}+\Delta_{2}\right)+\cdots .
\end{aligned}
$$

In the small angle approximation for the hyperbolic functions, this expression agrees with the linear stability condition for the filters drawn in Fig. 2.

The quadratic stability condition arises from terms where two of the matrices $U_{k} U_{m}$ are replaced by $t_{k}, t_{m}$ factors. For the three-cell case $N=3$, one has

$$
\begin{aligned}
& t_{1} t_{2} U_{3} Y\left(\mu_{X}\right)+t_{1} t_{3} U_{2} Y\left(\mu_{X}\right)+t_{2} t_{3} U_{1} Y\left(\mu_{X}\right) \\
&=t_{1} t_{2} \sinh \left(\mu_{3}-\mu_{X} / 2\right)+t_{1} t_{3} \sinh \left(\mu_{2}-\mu_{X} / 2\right) \\
&+t_{2} t_{3} \sinh \left(\mu_{1}-\mu_{X} / 2\right) \\
&= t_{1} t_{2} \sinh \left(\mu_{1}-\mu_{2}\right)+t_{1} t_{3} \sinh \left(\mu_{1}-2 \mu_{2}+\mu_{3}\right) \\
&+t_{2} t_{3} \sinh \left(\mu_{3}-\mu_{2}\right) \\
&= t_{1} t_{2} \sinh \left(-\Delta_{1}\right)+t_{1} t_{3} \sinh \left(\Delta_{2}-\Delta_{1}\right)+t_{2} t_{3} \sinh \left(\Delta_{2}\right) .
\end{aligned}
$$

TABLE II. Equations which determine the $\Delta_{k}$ for the $N=7$ filter, in the linear model.

\begin{tabular}{ccccccccc}
\hline \hline$\Delta_{0}$ & $\Delta_{1}$ & $\Delta_{2}$ & $\Delta_{3}$ & $\Delta_{4}$ & $\Delta_{5}$ & $\Delta_{6}$ & $\Delta_{7}$ & $=$ value \\
\hline 28 & -27 & 25 & -22 & 18 & -13 & 7 & 0 & 0 \\
0 & -27 & 75 & -132 & 180 & -195 & 147 & 0 & 0 \\
392 & -333 & 245 & -176 & 168 & -221 & 245 & 0 & 0 \\
0 & -333 & 705 & -792 & 600 & -585 & 1029 & 0 & 0 \\
13132 & -8028 & 4870 & -7018 & 7782 & -3562 & 11368 & 0 & 0 \\
0 & -669 & 630 & -319 & 875 & -130 & 1029 & 0 & 0 \\
1 & -1 & 1 & -1 & 1 & -1 & 1 & -1 & 0 \\
1 & 1 & 1 & 1 & 1 & 1 & 1 & 1 & $\mu_{X}$ \\
\hline \hline
\end{tabular}

It is easily seen that this vanishes for the binomial filter, providing that the $t_{k}$ are all equal. But for semiconductors where they are unequal, it is a new condition to be imposed on the $\Delta_{k}$.

The general $m$ th-order stability condition is worked out in Appendix A. It takes into account all terms where $m$ of the $t_{k}$ are involved, for $m=1,2, \ldots,(N-1)$.

\section{PRACTICAL APPLICATION OF STABILITY CONDITIONS}

For an $N$-cell impedance transformer, Eq. (20) expresses $M$ as a sum of $2^{N}$ terms, which fall into $N+1$ classes labeled by the number $m$ of $t_{k}$ occurring in the terms of class $m$. The matrix element $M_{21}$ must be zero for perfect transmission into the central cells $X$.

The vanishing of the $m=0$ term is expressed by $\mu_{\Sigma}$ $=0$ in Eq. (17). It involves just the alternating sum of the steps $\Delta_{k}, k=0 \cdots N$. The linear, quadratic, and higher stability conditions involve products of the $t_{k}$ times hyperbolic sinh's whose arguments are specific linear combinations of the $\Delta_{k}$. In the optical and microwave applications, the $t_{k}$ are the same for all $k$, so their value is just an overall factor that can be dropped from all terms in class $m$. In effect one can set $t_{k}=1$.

In semiconductors it is found that the strongest barrier has a $\cos \phi_{k}$ that varies most rapidly with energy, and the others vary progressively less rapidly. It will also be seen in the next section that in the vicinity of the multi-Bragg point, the $t_{k}$ vary linearly with energy. To the extent this is true we can replace the $t_{k}$ by their slopes, taking say the weakest one to be unity. A reasonable first approximation to this regime is to set $t_{k}=k$, which we will call the linear model. ${ }^{20}$ This contrasts with the situation in optics, where $t_{k}=$ constant applies, at least for normal incidence.

If we further make the small (hyperbolic) angle approximation, then the terms of class $m$ reduce to a linear combination of the unknowns $(-)^{k} \Delta_{k}$ multiplied by sums of products of the $t_{k}$. For example, for $N=3$, from Eqs. (17), (24), and (25) we can write

$$
\begin{aligned}
& \sum_{p} \Delta_{p}=\mu_{X}, \\
& \sum_{p}(-)^{p} \Delta_{p}=0, \\
& \left(t_{1}+t_{2}+t_{3}\right) \Delta_{0}-\left(t_{2}+t_{3}\right) \Delta_{1}+t_{3} \Delta_{2}=0, \\
& -\left(t_{1} t_{2}+t_{1} t_{3}\right) \Delta_{1}+\left(t_{1} t_{3}+t_{2} t_{3}\right) \Delta_{2}=0 .
\end{aligned}
$$


TABLE III. Linear model solutions $\Delta_{k}$ for the $N=7$ filter.

\begin{tabular}{ccccc}
\hline \hline$k=$ & $\Delta_{k}$ & $\mu_{k+1}$ & Poisson $_{k}$ & $\mu_{\text {Poisson }}$ \\
\hline 0 & 0.125 & 0.125 & 0.166 & 0.166 \\
1 & 0.2917 & 0.4167 & 0.298 & 0.464 \\
2 & 0.2917 & 0.7083 & 0.268 & 0.732 \\
3 & 0.1856 & 0.8939 & 0.160 & 0.892 \\
4 & 0.07954 & 0.9735 & 0.072 & 0.964 \\
5 & 0.02244 & 0.9959 & 0.0259 & 0.990 \\
6 & 0.00379 & 0.9997 & 0.0077 & 0.998 \\
7 & 0.000291 & 1 & 0.0002 & 0.998 \\
\hline \hline
\end{tabular}

It is convenient to take $D_{p}=(-)^{p} \Delta_{p}$ as the unknowns; this removes most of the negative signs from the equations. Leaving aside the top equation, which gives the overall normalization of the solution, a bit of algebra puts the remaining three into the form

$$
\left(\begin{array}{cccc}
1 & 1 & 1 & 1 \\
0 & x_{1} & x_{2} & x_{3} \\
0 & x_{1}^{2} & x_{2}^{2} & x_{3}^{2}
\end{array}\right)\left(\begin{array}{c}
D_{0} \\
D_{1} \\
D_{2} \\
D_{3}
\end{array}\right)=0
$$

where $x_{k}=t_{1}+\cdots+t_{k}$ is a partial sum of the slopes. We can solve these equations for $D_{1}, D_{2}, D_{3}$ in terms of $D_{0}$ and then use the top equation of Eqs. (26) to give the normalized solutions. In this case (but not for larger $N$ ), the coefficient matrix is equivalent to the well-known Vandermonde matrix, and the solution can be written down immediately. ${ }^{23}$

In general, the stability conditions of order $m$ $=1,2, \ldots,(N-1)$, together with Eqs. (17) become a set of $N+1$ linear equations for the unknowns $\Delta_{k}$, and a similar strategy can be used to solve them. An example is shown in Table I, for $N=7$, where we have set all the $t_{k}$ equal to unity, appropriate for optics. As expected, the solution is the binomial filter for $N=7$. In contrast, in Table II we show the equations for the linear model, with $t_{k}=k$. The solution, shown in Table III is now completely different. The first few steps in $\mu_{k}$ are much larger than in the binomial filter, and the last few steps are very much smaller. The reason is that the relatively rapid variation of the last few Bloch phases can only be countered by making the radii of the circles of (spin)

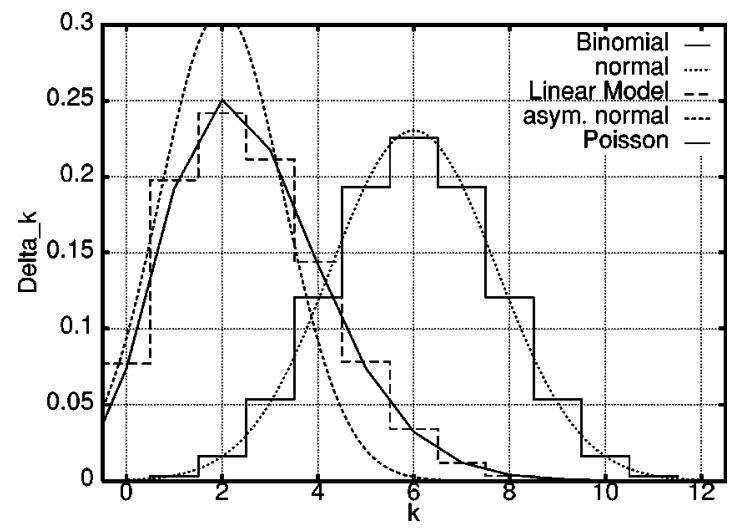

FIG. 3. Steps $\Delta_{k}$ for binomial and linear models, compared to Gaussian and Poisson distributions, for 12 cell ARC.
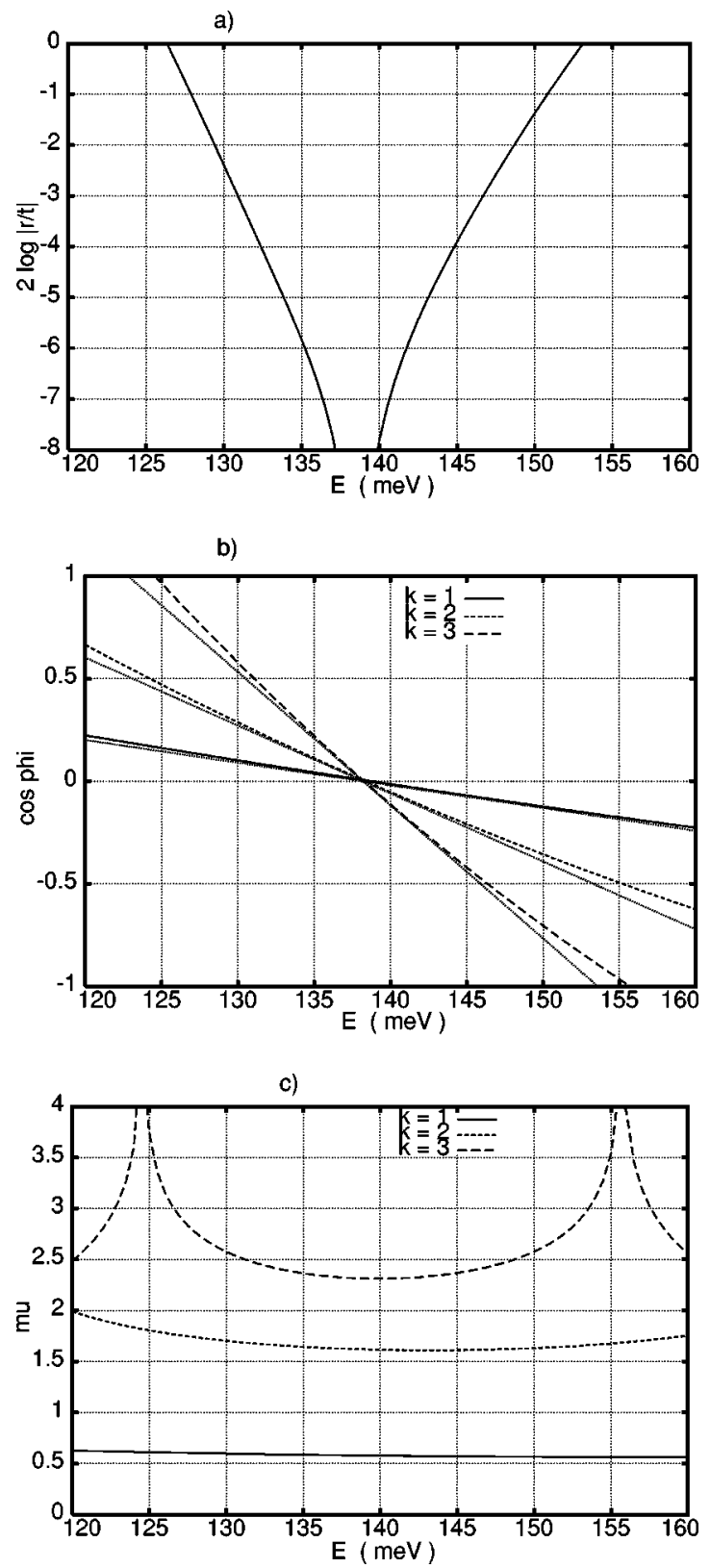

FIG. 4. Analysis of the (adjusted) six-cell device of Coquelin et al. (a) $\log |r / t|^{2}$, (b) $\cos \phi_{k}$, and their tangents at the band center, and (c) $\mu_{k} \cdot{ }^{21}$

rotation as small as possible. The structure of a filter for semiconductors therefore will have a very different profile than for optical or microwave applications.

This difference in character is illustrated in Fig. 3, for a 12-cell ARC filter. The steps $\Delta_{k}$ for the binomial filter are peaked at $k=6$, and are well fitted by a Gaussian distribution. In contrast, for the linear model, the steps (at left) peak at $k=2$ and are better represented by a Poisson distribution than by an asymmetric Gaussian having the same average value.

The general solution of the linear model for $N$ cells is

$$
\begin{aligned}
\Delta_{p} & =(2 p+1) \frac{N !}{(N-p) !} \frac{N !}{(N+p+1) !} \\
& =\frac{2 p+1}{N+p+1} \frac{{ }_{N} C_{p}}{{ }_{N+p} C_{p}}, \quad p=0 \cdots N .
\end{aligned}
$$




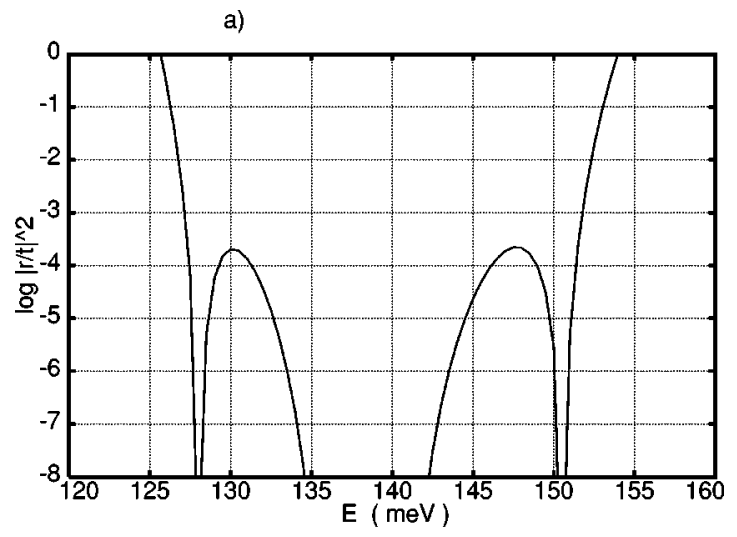

b)

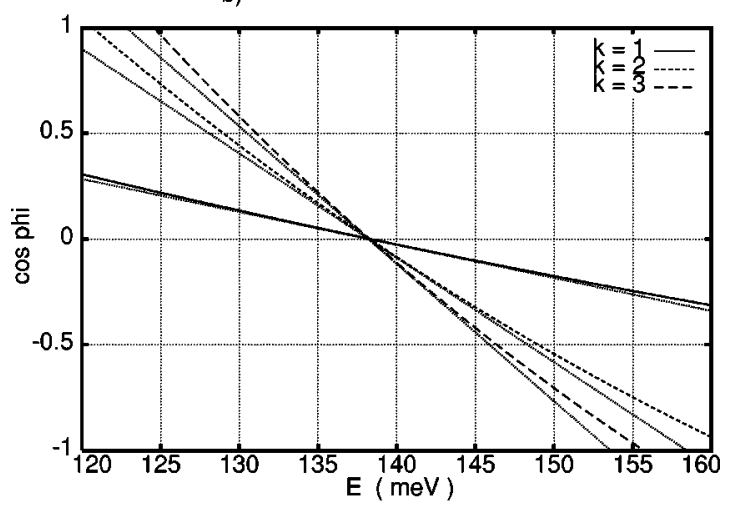

c)

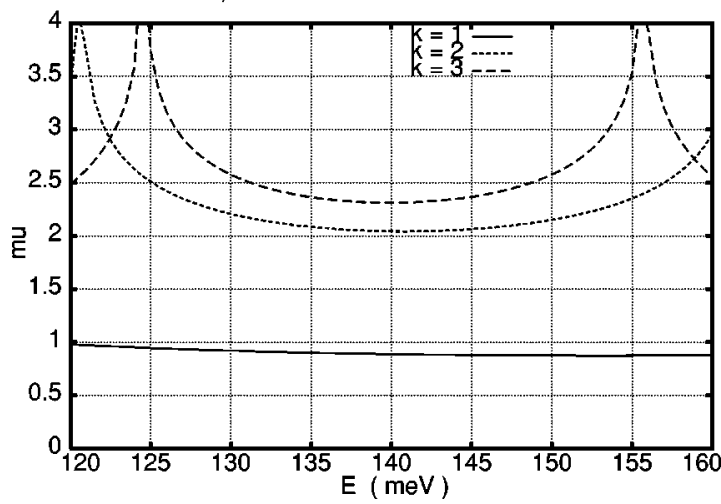

FIG. 5. Analysis of the self-consistent $N=2$ ARC device derived from Coquelin et al. (a) $\log |r / t|^{2}$, (b) $\cos \phi_{k}$, and their tangents at the band center, and (c) $\mu_{k} \cdot{ }^{21}$

For large $p \approx N$ the linear model steps are exponentially small. Correspondingly, $\Delta_{0}=1 /(N+1)$, which for $N>4$ greatly exceeds the value for the binomial or Butterworth filter, $2^{-N}$. The Poisson distribution $P_{k}=a^{k} e^{-a} / k$ ! can be fitted by setting $\Delta_{0}=e^{-a}$ to fix the mean value $a$. Alternatively we can choose $a$ to give the second moment of the distribution of steps, as was done in the figure.

One conclusion we can draw is that a semiconductor bandpass filter should have many fewer cells than a similar optical or microwave filter. Most of the work will be done by the first few cells, so the later ones contribute less to the performance. This is useful information because it is easier to make a semiconductor device with fewer cells. a) $E=134 \mathrm{meV}$

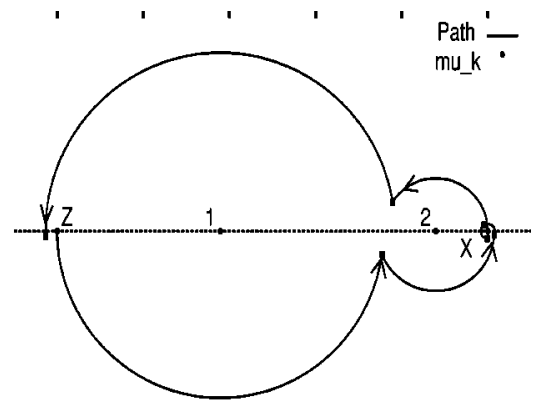

b) $E=140 \mathrm{meV}$
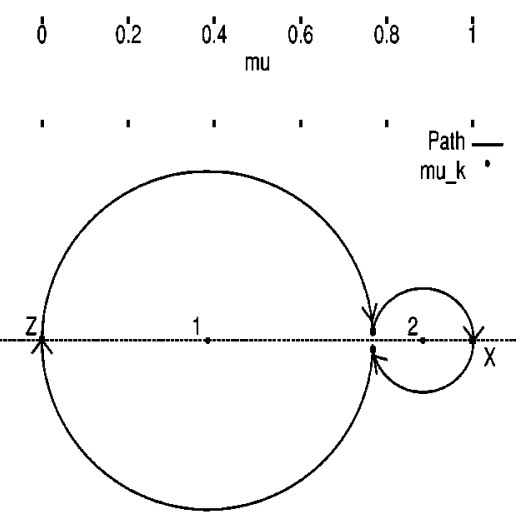

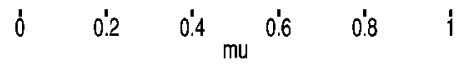

c) $E=150 \mathrm{meV}$

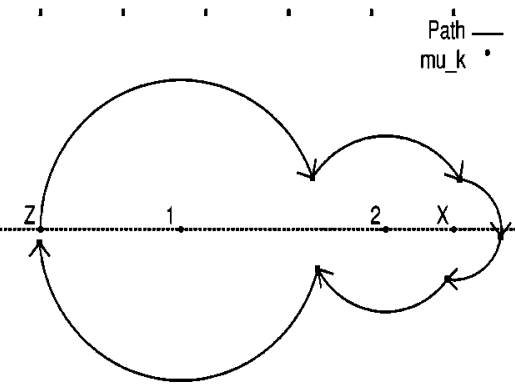

$0 \quad 0^{1} 2 \quad 0^{1} 4 \mathrm{mu}^{0.6} \quad 0^{1} .8 \quad 1$

FIG. 6. Pseudospin trajectories for the self-consistent $N=2$ device at three energies as labeled.

\section{EXAMPLE}

On the web site of Gornik's group in Vienna, Coquelin et al. ${ }^{8}$ presented an example of a six-barrier system which achieves $83 \%$ transmissivity over the first allowed miniband. The structure was modeled as a sequence of $\mathrm{Al}_{x} \mathrm{Ga}_{1-x} \mathrm{As}$ quantum barriers with $x=0.3$, and GaAs wells. Viewed as a one-dimensional (1D) potential array, the barrier heights are $290 \mathrm{meV}$, and the widths were varied according to a Gaussian law, ${ }^{14}$ taking the values 9,28 , and $40 \AA$. The well widths were set at $30 \AA$.

We can consider this an example of an ARC system with $N=2$ cells, surrounding two central cells $X$. We define a cell to consist of one barrier and a $15 \AA$ spacer on each side of it. In our calculation, we took the effective mass at the conductance band edge to be 0.092 and 0.067 , respectively, and the energy gaps 1800 and $1424 \mathrm{MeV}$, based on Davies. ${ }^{22} \mathrm{We}$ took account of energy dependence of the effective masses 

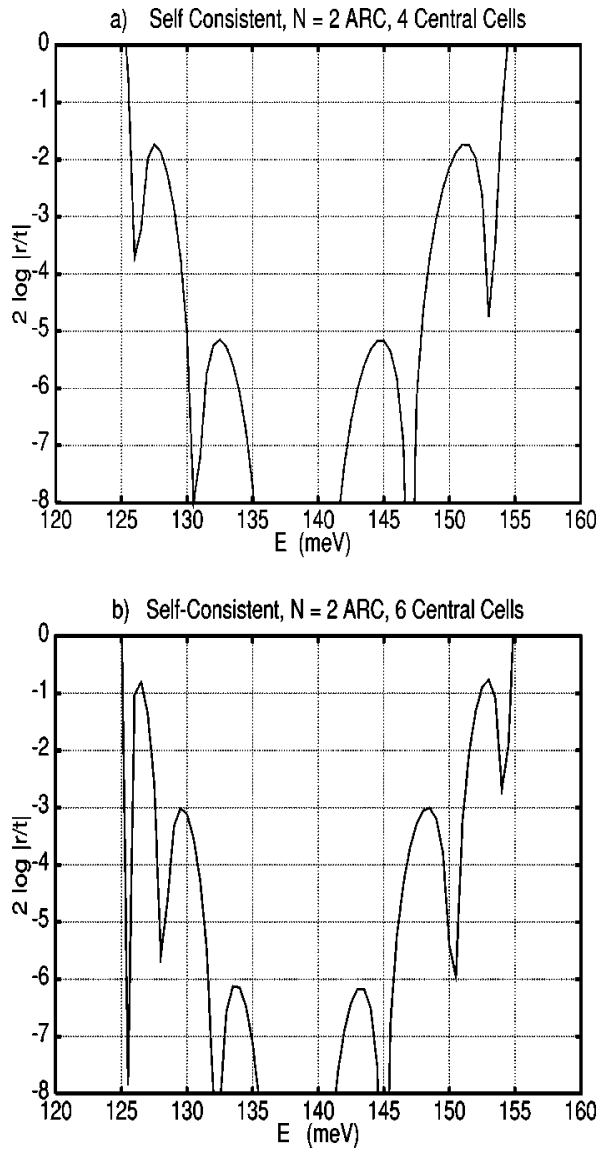

FIG. 7. Logarithmic plot of $|r / t|^{2}$ for $N=2$ ARC, with four and six central cells, self-consistent solution.

following the recipe of Nelson et al. ${ }^{16}$ Finally, following Pacher et al. ${ }^{7}$ we took the barrier height to be $290 \mathrm{MeV}$. As a check of these parameters, we reproduced the five peaks shown between 128 and $160 \mathrm{meV}$ in the figure of Ref. 8 for a periodic six-cell array.

Computing the Bloch phases and impedance parameters for each cell of their Gaussian array, one finds that every cell is a Bragg reflector close to the band center. The multi-Bragg character was improved by using $10 \AA$ for the width of the weakest barrier and $15.05 \AA$ for the corresponding half-well; with these small adjustments, the common Bragg point is at $138.3 \mathrm{meV}$. We will refer to this as the "adjusted" Coquelin array. The performance is illustrated in Fig. 4(a), where we plot $|r / t|^{2}$ for the entire device, on a natural $\log$ scale. This is a more sensitive presentation than simply plotting the transmission probability which would merely show a flat band with values at $|t|^{2}=1$.

The $\mu_{p}$ are plotted ${ }^{21}$ in Fig. 4(c); at the Bragg point they take values $0.580: 1.623: 2.319$ which are very nearly in the ratio 1:3:4 of a classic Butterworth filter. However, moving away from the multi-Bragg point, the slopes of the $\cos \phi_{p}$ lines, seen in Fig. 4(b), are in the ratio 0.17:0.51:1.0 which is very far from equal slopes; indeed, rather close to the linear model.

Taking the parameters $t_{p}$ to be the above-mentioned slopes, and solving the stability conditions predicts that the $\mu_{p}$ should be close to those of the linear model, which are
TABLE IV. Barrier widths, $t_{p}$ and $\mu_{p}$ for $N$-cell ARCs, both self-consistent and modified fits.

\begin{tabular}{|c|c|c|c|c|c|c|}
\hline$N$ & Type & $\begin{array}{l}b_{1} \\
t_{1} \\
\mu_{1}\end{array}$ & $\begin{array}{l}b_{2} \\
t_{2} \\
\mu_{2}\end{array}$ & $\begin{array}{l}b_{3} \\
t_{3} \\
\mu_{3}\end{array}$ & $\begin{array}{l}b_{4} \\
t_{4} \\
\mu_{4}\end{array}$ & $b_{X}$ \\
\hline \multirow[t]{3}{*}{2} & adj. & 10.0 & 28.0 & & & 40 \\
\hline & & 0.17 & 0.51 & & & \\
\hline & 0.828 & 0.580 & 1.623 & & & 2.319 \\
\hline \multirow[t]{3}{*}{2} & s-c & 15.4 & 35.4 & & & 40 \\
\hline & & 0.24 & 0.76 & & & \\
\hline & 0.902 & 0.893 & 2.052 & & & 2.319 \\
\hline \multirow[t]{3}{*}{2} & $\bmod$ & 16.4 & 35.3 & & & 40 \\
\hline & & 0.25 & 0.73 & & & \\
\hline & 0.920 & 0.951 & 2.047 & & & 2.319 \\
\hline \multirow[t]{3}{*}{3} & s-c & 12.4 & 31.1 & 38.6 & & 40 \\
\hline & & 0.21 & 0.64 & 1 & & \\
\hline & 0.942 & 0.719 & 1.803 & 2.238 & & 2.319 \\
\hline \multirow[t]{3}{*}{3} & $\bmod$ & 12.4 & 30.4 & 38.2 & & 40 \\
\hline & & 0.21 & 0.63 & 1 & & \\
\hline & 0.944 & 0.719 & 1.763 & 2.215 & & 2.319 \\
\hline \multirow[t]{3}{*}{4} & $\mathrm{~s}-\mathrm{c}$ & 10.3 & 27.6 & 36.6 & 39.3 & 40 \\
\hline & & 0.18 & 0.50 & 0.84 & 1 & \\
\hline & 0.962 & 0.597 & 1.600 & 2.122 & 2.279 & 2.319 \\
\hline \multirow[t]{3}{*}{4} & $\bmod$ & 10.3 & 27.5 & 36.3 & 39.2 & 40 \\
\hline & & 0.18 & 0.50 & 0.84 & 1 & \\
\hline & 0.964 & 0.597 & 1.594 & 2.105 & 2.273 & 2.319 \\
\hline
\end{tabular}

$1 / 3,5 / 6,1$ times $\mu_{X}$. This suggests that improved performance should result from changing the barrier widths, to produce impedances which are consistent with the ensuing slopes.

Because the barriers are relatively high, the $\mu_{p}$ are roughly proportional to the widths. Keeping the central cell parameters fixed $\left(b_{X}=40 \AA\right)$, we found that with $b_{1}$ $=15.4 \AA$ and $b_{2}=35.4 \AA$, the resulting $\mu_{p}$ took values $0.893,2.052$, compared to $\mu_{X}=2.319$, which are selfconsistent with the solution of the stability equations using $t_{1}=0.24, t_{2}=0.76$. In Fig. 5(c) we show the $\mu_{k}(E)$ across the allowed band. The values are quite flat in the center of the band, though all three curve upwards as the band edge is approached. It is not necessary for the $\mu_{k}$ to be strictly constant; if their ratios are constant the spin analogy shows that the ARC mechanism will still work. The $\cos \phi_{p}$ are shown in Fig. 5(b) along with the linear approximation from which the $t_{p}$ were estimated. In Fig. 5(a) we show, on a log scale, $|r / t|^{2}$ for this solution. This is to be compared with Fig. 4(a), which used the (adjusted) Coquelin parameters. The average transmissivity over the band $\tau$ has increased from 0.83 to 0.90 .

In Fig. 6 we show the trajectories of the spin analogy, at three energies. In the first two cases (near the band center) the spin orientation has indeed been moved to the point $\mu_{X}$, and then back to the origin. This confirms that linear stability has been satisfied for the self-consistent filter. The third panel (c) is at $150 \mathrm{meV}$, in a deep trough of $|r / t|^{2}$. The performance is degrading and is only maintained because the ARC cells bring the spin to a point below $\mu_{X}$, and the two central cells bring it (almost) to the mirror image point above the axis. Then the downstream ARC cells can bring it back to OZ. 

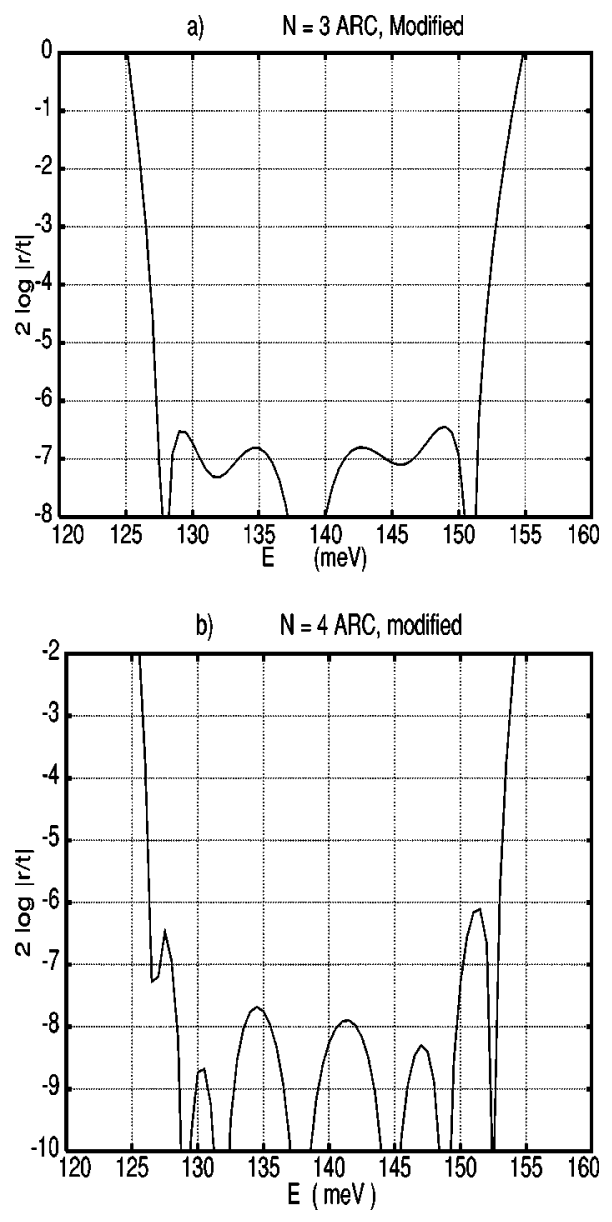

FIG. 8. Logarithmic plot of $|r / t|^{2}$ for $N=3$ and $N=4$ ARCs, with parameters optimized.

The performance should not depend on the number of central cells. This is true close to the design energy, but not further away, as seen in the previous paragraph. In Figs. 7(a) and 7(b) we show $\log |r / t|^{2}$ for the same ARC with $K=4$ and $K=6$ central cells. Additional humps are seen, the number increasing like $K$, but always lying below some upper limit. This limit is related to the envelope of transmission minima, as discussed in Ref. 9. The width of the region with good performance widens slightly as $K$ increases. Mostly this is the expected effect of the band edges becoming better defined by the central periodic structure. The transmissivity rises from 0.902 to $0.912(K=4)$ and $0.915(K=6)$, using the self-consistent $N=2$ parameters.

Some further improvement can be obtained by small variations in the parameters. Basically this involves a tradeoff, making the inside humps of $|r / t|^{2}$ a little higher and the outlying ones a little lower. One gains a bit on the width of the region of low reflectivity, while keeping the value under some maximum, say $e^{-5}$. Our theory is based on assuming constant $\mu_{p}$ and $t_{p}$, but both of these break down as you move away from the multi-Bragg point. The small adjustments gain on the edges at the price of not hitting the target at the Bragg point. (In optics or microwaves, such a fit is referred to as a Chebyshev filter. In our case of unequal $\phi_{p}$, one cannot use the Chebyshev polynomials, but rather the intent, which is to widen the passband while maintaining a maximum value on the reflectance.) The best fit does depend on $K$ because the humps that are being reduced have locations which depend on $K$.

We conclude that the ARC obtained from solving the stability equations has improved the filter performance by about $10 \%$, or close to half the gap from ideal performance, even for $N=2$ cells.

Further improvement is obtained by using a three- or four-cell ARC. The parameters of these ARC solutions are shown in Table IV. The barrier widths are in $\AA$, while the other values are dimensionless. In all cases the well widths are $30 \AA$, except for the weakest barrier where 30.05 is maintained. The difference between the self-consistent and modified solutions is always small, but there is a gain in average transmissivity.

In Fig. 8 we show plots of $\log |r / t|^{2}$ for the modified solutions. They show that the filter bandwidth has increased as compared to the $N=2$ filter. Note the change of vertical scale between panels (a) and (b) by a factor $e^{-2}$.

\section{CONCLUSION}

We have used the analogy between potential scattering and precession of a spin-half system, to provide a simple and intuitive picture of the workings of a quarter-wave impedance transformer or passband filter. Based on this picture we have identified the stability conditions which take into account the different rates of variation of the $\cos \phi_{p}$ with energy. Enforcing these conditions makes an $N$ th order zero of the reflection amplitude for a system with an $N$-cell antireflection coating. The rules for writing down these conditions are given in Appendix A.

Under the small-angle (hyperbolic) approximation, the stability conditions become a set of $N+1$ linear equations for the impedance steps $\Delta_{p}$. When the Bloch phases of the cells vary at the same rate, as in optical ARC's, the solution of these equations is the well-known binomial filter, Eq. (19). For semiconductor superlattices, the rate of variation of the Bloch phases depends strongly on the strength of the potential cell. A reasonable first approximation is provided by the linear model, in which $t_{p}=p$. We have given the exact solution of the linear model in Eq. (28). As seen in Fig. 3, the steps of the linear model are close to a Poisson distribution, completely different from the Gaussian limit of the binomial distribution.

For arbitrary values of the $t_{p}$, the stability conditions can be computed and the linear equations solved for the corresponding $\Delta_{p}$. A numerical strategy for computing the coefficient matrix is outlined in Appendix B. This allows for an iterative approach to the design of impedance filters. We have illustrated this process by finding a system similar to that of Coquelin et al. Their system was shown to be a Butterworth filter. By adjusting it to satisfy the stability conditions we improved the average transmissivity from 0.83 to 0.90 over the allowed band. Increasing the number of ARC cells provides further improvement. 


\section{ACKNOWLEDGMENTS}

We are grateful to NSERC-Canada for discovery Grant No. SAPIN-3198 (DWLS, GVM) and to DGES-Spain for continued support through Grant Nos. PB97-0915 and BFM2001-3710 (JM). We also thank Gideon Humphrey, holder of an NSERC SRA, for assistance with the calculations and drawings. This work was carried out as part of CERION, Esprit project EP-27119 funded by the EU and NSERC.

\section{APPENDIX A: GENERAL STABILITY CONDITION}

The stability condition of order $m$ includes terms where some set $k=k_{1}, k_{2}, \ldots, k_{m}$ of the $t_{k}$ 's replace the corresponding factors $U_{k}$ in the expansion Eq. (20). We define the resulting 1,2 matrix element contribution of this term as $\sinh \chi\left(k_{1}, k_{2}, \ldots, k_{m}\right)$, times the product of the corresponding $t_{k}$, and with the requisite number of factors of $-i$. The case $m=1$ was treated in full in the main text, as was $m=2$. Learning from these examples we can state the general rule: The argument $\chi\left(k_{1}, k_{2}, \ldots, k_{m}\right)$ involves a sum of the $(-)^{p} \Delta_{p}$, each with its proper sign. If $m$ is even, then the included terms are those between $k_{1}$ and $k_{2}-1$, then from $k_{3}$ to $k_{4}-1$, etc. When $m$ is odd, however, the first sum runs from $p=0$ to $k_{1}-1$, and then successive groups run from $k_{2}$ to $k_{3}-1$, etc. In every case the last group ends at $k_{m}-1$. The reason is that removal of any of the $U_{p}$ matrices causes a glitch in the progression of signs, and with an odd number of glitches, the final entry $\mu_{X}$ will occur with the "wrong" sign. Then we must add $\mu_{\Sigma}$ to remove $\mu_{X}$ from the phase. Conversely, with an even number of glitches we must subtract $\mu_{\Sigma}$. Addition causes the first group of entries to be included in $\chi$ while subtraction removes them.

We can now write down the general term in the expansion of the transfer matrix for $N$ cells. The leading term is the product of $\sin \phi_{p}$ times $\sinh \mu_{\Sigma}$. [See Eq. (20).] Dropping an overall phase, this contributes to the real part of $r / t$ for the system. The $m=1$ terms, Eq. (24), contribute to the imaginary part of $r / t$. In them, one of the sines is changed into a cosine; which is accounted for by the factor $t_{p}$. In general, the odd-order corrections contribute to the imaginary part and the even order ones to the real part (or vice versa depending on the parity of $N$ ). There are altogether $N$ such correction types, which we call the stability conditions. Along with the normalizing condition $\Sigma \Delta_{p}=\mu_{X}$, they are sufficient to determine the values of the $\Delta_{p}(p=0,1, \ldots$, $N-1$ ). This solution is valid over a range of energies where the $t_{p}$ vary linearly with energy around the multi-Bragg point. This allows the design of a generalized Butterworth transformer for application to electrons in semiconductors.

\section{APPENDIX B: PROGRAMMING STABILITY EQUATIONS}

The expansion Eq. (20) contains $2^{N}$ terms, which are in $(1,1)$ correspondence with the binary integers $j$ $=\left[b_{N} b_{N-1} \cdots b_{1}\right]$. (The $b_{q}$ are binary bits.) Those $j$ which have $m$ nonzero bits contribute to the $m$ th stability equation. For each $b_{k}=1$, a factor $t_{k}$ is included in the coefficient.
We make the small-angle approximation $\sinh \chi \approx \chi$. The stability equations reduce to the form

$$
\sum_{p=0}^{N-1} C_{p}^{(m)}(-)^{p} \Delta_{p}=0, \quad m=0 \cdots(N-1) .
$$

Starting with $j=0$, up to $2^{N}-1$, each binary integer is parsed, assigned to class $m$, and the argument $\chi\left(k_{1}, k_{2}, \ldots, k_{m}\right)$ is constructed as stated in Appendix A. Then for each of the $\Delta_{p}$ occurring in $\chi$, the coefficient $C_{p}^{(m)}$ is augmented by the product of $t_{k}$, with $k=k_{1}, \ldots, k_{m}$. In this way the $N-1$ stability equations can be constructed using only $N^{2}$ storage locations.

The $j=0$ term corresponds to the basic equation Eq. (17) for $\mu_{\Sigma}=0$. As $m$ ranges over the values $0,1, \ldots$, $N-1$, we obtain $N$ such equations, in the $N+1$ unknowns $\Delta_{p}$. They are supplemented by the second equation in Eq. (17), which normalizes the sum of the $\Delta$ 's to $\mu_{X}$, and makes the system soluble. $\Delta_{N}$ occurs only in this extra equation, so one strategy is to solve the stability equations for the $\Delta_{p}$, $p=0,1, \ldots, N-1$ in terms of $\Delta_{N}$, and then use the last equation to complete the solution.

Obviously, as $N$ increases, the time taken to accumulate the $2^{N}$ contributions to the coefficients increases exponentially. Our code, written in $\mathrm{C}++$, works well up to $N$ $=24$. The method is general but for larger $N$ one needs to use higher-precision integer representation for $j$. Fortunately, for the binomial and linear models, we have analytic solutions for general $N$, and these can be used to check the computer program.

${ }^{1}$ R. J. Pegis, J. Opt. Soc. Am. 51, 1255 (1961).

${ }^{2}$ E. Delano and R. J. Pegis, Prog. Opt. 7, 69 (1969).

${ }^{3}$ P. G. Kard, Opt. Spektrosk. 2, 236 (1957).

${ }^{4}$ H. J. Riblet, IRE Trans. Microwave Theory Tech. 5, 36 (1957).

${ }^{5}$ R. E. Collin, Proc. IRE 43, 176 (1955).

${ }^{6}$ R. E. Collin, Foundations of Microwave Engineering (McGraw-Hill, New York, 1966).

${ }^{7}$ C. Pacher, C. Rauch, G. Strasser, E. Gornik, F. Elsholz, A. Wacker, G. Kiesslich, and E. Schöll, Appl. Phys. Lett. 79, 1486 (2001). See also C. Pacher et al., Photonics Spectra 12, 285 (2001).

${ }^{8}$ M. Coquelin, M. Kast, C. Pacher, G. Fasching, G. Strasser, and E. Gornik, Tech. Univ. Vienna (unpublished); 〈www.fke.tuwien.ac.at/transport/ transport.htm $\rangle$.

${ }^{9}$ G. V. Morozov, D. W. L. Sprung, and J. Martorell, J. Phys. D 35, 2091 (2002); 35, 3052 (2002)

${ }^{10}$ T. K. Gaylord, E. N. Glytsis, and K. F. Brennan, J. Appl. Phys. 65, 2535 (1989).

${ }^{11}$ T. K. Gaylord, E. N. Glytsis, and K. F. Brennan, J. Appl. Phys. 67, 2623 (1990).

${ }^{12}$ C. C. Chang and C. S. Kuo, J. Phys. D 32, 139 (1999).

${ }^{13}$ H. H. Tung and C. P. Lee, IEEE J. Quantum Electron. 32, 507 (1996); 32, 2122 (1996).

${ }^{14}$ I. Gomez, F. Dominguez-Adame, E. Diez, and V. Bellani, J. Appl. Phys. 85, 3916 (1999).

${ }^{15}$ Q. K. Yang and A. Z. Li, J. Appl. Phys. 87, 1963 (2000).

${ }^{16}$ D. F. Nelson, R. C. Miller, and D. A. Kleinman, Phys. Rev. B 35, 7770 (1987).

${ }^{17}$ At a band edge, $\mu$ diverges in such a way that $-i \sin \phi \sinh \mu=M_{21}$ has the correct value. A similar form for the transfer matrix holds in a forbidden band. Both $\phi$ and $\mu$ vary continuously across the band edge, where they become complex.

${ }^{18}$ J. J. Sakurai, in Modern Quantum Mechanics, revised edition, edited by S. F. Tuan (Addison-Wesley, Reading, MA, 1994), Chap. 3. 
${ }^{19}$ D. W. L. Sprung, Hua Wu, and J. Martorell, Am. J. Phys. 61, 1118 (1993).

${ }^{20}$ The Linear model is a rough approximation to the detailed behavior of the $\cos \phi_{k}$, which captures the dominant trend that the phases for the stronger barriers vary much more rapidly than those of the weaker ones. It has the advantage of an analytical solution, from which one can draw general conclusions. In optics the corresponding approximation of constant indices of refraction leads to the binomial filter design.
${ }^{21}$ In a forbidden band, $\mu_{k} \rightarrow \xi_{k} \pm i \pi / 2$; the disjoint line segments below 125 $\mathrm{meV}$ and above $156 \mathrm{meV}$ are $\xi_{k}$.

${ }^{22}$ John H. Davies, Physics of Low-dimensional Semiconductors (Cambridge University Press, Cambridge, England, 1998).

${ }^{23}$ W. H. Press, S. A. Teutolksky, W. T. Vetterling, and B. P. Flamery, Numerical Recipes in C (Cambridge University Press, Cambridge, England, 1993), Sec. 2.8. 